\title{
Tumor Suppressor Gene-Based Nanotherapy: From Test Tube to the Clinic
}

\author{
Manish Shanker, ${ }^{1}$ Jiankang Jin, ${ }^{1}$ Cynthia D. Branch, ${ }^{1}$ Shinya Miyamoto, ${ }^{2}$ \\ Elizabeth A. Grimm, ${ }^{3}$ Jack A. Roth, ${ }^{1}$ and Rajagopal Ramesh ${ }^{1,2,4,5}$ \\ ${ }^{1}$ Department of Thoracic and Cardiovascular Surgery, The University of Texas of MD Anderson Cancer Center, Houston, \\ TX 77030, USA \\ ${ }^{2}$ Department of Pathology, The University of Oklahoma Health Sciences Center, Oklahoma City, OK 73104, USA \\ ${ }^{3}$ Department of Experimental Therapeutics, The University of Texas of MD Anderson Cancer Center, Houston, TX 77030, USA \\ ${ }^{4}$ Peggy and Charles Stephenson Oklahoma Cancer Center, The University of Oklahoma Health Sciences Center, Oklahoma City, \\ OK 73104, USA \\ ${ }^{5}$ Graduate Program in Biological Sciences, The University of Oklahoma Health Sciences Center, Oklahoma City, OK 73104, USA
}

Correspondence should be addressed to Rajagopal Ramesh, rajagopal-ramesh@ouhsc.edu

Received 6 October 2010; Accepted 5 November 2010

Academic Editor: Sanyog Jain

Copyright (C 2011 Manish Shanker et al. This is an open access article distributed under the Creative Commons Attribution License, which permits unrestricted use, distribution, and reproduction in any medium, provided the original work is properly cited.

\begin{abstract}
Cancer is a major health problem in the world. Advances made in cancer therapy have improved the survival of patients in certain types of cancer. However, the overall five-year survival has not significantly improved in the majority of cancer types. Major challenges encountered in having effective cancer therapy are development of drug resistance by the tumor cells, nonspecific cytotoxicity, and inability to affect metastatic tumors by the chemodrugs. Overcoming these challenges requires development and testing of novel therapies. One attractive cancer therapeutic approach is cancer gene therapy. Several laboratories including the authors' laboratory have been investigating nonviral formulations for delivering therapeutic genes as a mode for effective cancer therapy. In this paper the authors will summarize their experience in the development and testing of a cationic lipid-based nanocarrier formulation and the results from their preclinical studies leading to a Phase I clinical trial for nonsmall cell lung cancer. Their nanocarrier formulation containing therapeutic genes such as tumor suppressor genes when administered intravenously effectively controls metastatic tumor growth. Additional Phase I clinical trials based on the results of their nanocarrier formulation have been initiated or proposed for treatment of cancer of the breast, ovary, pancreas, and metastatic melanoma, and will be discussed.
\end{abstract}

\section{Introduction}

Cancer is a major health problem in the world. In 2009, about 1,479,350 people living in the United States of America (USA), have been diagnosed with cancer [1]. About half of these cancer patients will die of the disease. The lifetime risk of developing cancer is predicted to be 1 in 2 for men and 1 in 3 for women [1]. Dissemination of scientific information and cancer awareness have reduced the incidence for certain cancer types while the incidence for other cancer types remain unchanged or increased. For example, reduced incidence of lung cancer in men due to cessation of smoking has been observed while the lung cancer incidence in women is increasing. Similarly, ignoring the risks of exposure to ultraviolet rays and the potential for developing skin cancer has resulted in steady increase in the incidence of melanoma.

Effective cancer therapies developed in recent years have improved the survival of patients diagnosed with cancer. However, the overall five-year survival rate of cancer patients remain dismal and is less than $15 \%$ at least for solid tumors of epithelial origin [2]. Factors contributing to the poor survival rate despite having developed novel therapies include development of resistance to therapy by cancer cells, poor drug distribution and accumulation in the tumor, and nonspecific cytotoxicity to normal tissues thereby limiting the drug dosage. Thus, there is a tremendous effort in 
developing new cancer therapeutics that are efficacious and safe with minimal cytotoxicity to normal tissues. Testing and demonstration of such new therapeutics in preclinical studies will ultimately lead to testing in humans as a cancer drug.

One therapeutic approach that has shown promise and safety is cancer gene therapy [3]. The gene therapy approach that has exploded and tested widely in the last decade is the use of tumor suppressor genes (TSG's). Cell division and cell growth are tightly controlled processes often regulated by TSG's. However, alterations such as mutations, deletions, and silencing at the DNA, RNA, or protein level of TSG result in dysregulation of the cell growth and transformation [4]. Retinoblastoma (Rb) and p53 TSG are classical examples whose function when lost or altered has been shown to initiate or contribute to cell transformation $[5,6]$. Furthermore, p53 gene mutations are observed in a majority of human cancers, suggesting it is an important gatekeeper of the cell. Apart from Rb and p53, several other TSGs have been identified and shown to regulate diverse cellular processes and loss of their function affects normal cell activity. Based on these observations, it was hypothesized that restoration of normal TSG function will inhibit cell proliferation and growth leading to cell death. Thus TSG-based cancer therapy was conceived and initiated.

Early studies using viral vectors demonstrated that delivering TSG's resulted in tumor inhibition in animal models [3] (see Table 1). Translating these findings to the clinic demonstrated clinical and/or biological response to therapy. Stabilization of the disease (SD) was frequently observed in patients receiving therapy, and in few cases complete response to therapy as evidenced by tumors' regression [7-10]. Despite the encouraging clinical results observed in virus-based cancer gene therapy studies, this treatment strategy has limited application due to the elicitation of hostimmune response by viral proteins [11-14]. Additionally, testing of virus-based cancer gene therapy for treatment for metastatic disease has not been proven to be successful so far.

To overcome the limitations encountered with virusbased cancer therapy, several laboratories including our own laboratory have been testing nonviral-gene-delivery vehicles for cancer gene therapy. The nonviral vectors are of different composition and formulations. They also vary in their size and geometry. A majority of these nonviral vectors are nanometer $(\mathrm{nm})$ in size and often have a lipid component. According to the National Cancer Institute (NCI), any biological or synthetic material which in any one dimension is less than 1 micrometer $(\mu \mathrm{m})$ is called a nanoparticle. Based on this definition, several nonviral vectors that are less than $1 \mu \mathrm{m}$ in size are referred as nanoparticles, nanocarriers, nanosomes, and so forth.

An advantage of using nanoparticles as gene-delivery vehicles is that they can deliver therapeutic genes to in situ tumors that are disseminated inside the body [3,15]. Studies have demonstrated nanoparticle-based gene-delivery results in antitumor activity in experimental preclinical tumor models. An added advantage of using nonviral nanocarrier systems, apart from the ease of manufacturing, is the avoidance of problems frequently encountered with adenovirus $[15,16]$.
In this paper, we will discuss our experiences with a lipidbased nanocarrier that was initially tested in the laboratory as a tumor suppressor gene-delivery vehicle and later tested in the clinic for the treatment of nonsmall cell lung cancer (NSCLC). Plans for applying our nanocarrier-based cancer gene therapy technology for treatment of other solid cancers will also be discussed.

\section{Gene-Based Nanotherapy}

2.1. Laboratory Studies. Our interest in testing lipid-based nanocarriers as gene-delivery vehicles arises from the following observations: (1) cancer is often metastasized in patients at the time of their initial diagnosis $[1,2]$; (2) conventional therapies are ineffective in treating metastatic disease [17, 18]; (3) our own laboratory studies demonstrate that virus-based (retrovirus and adenovirus) tumor suppressor gene therapy for systemic therapy of metastatic cancer was ineffective; (4) preclinical studies demonstrated that nonviral vectors can deliver genes and drugs to localized and disseminated tumors [19-21].

Although several lipid-based nanocarriers were reported in the literature to be efficient gene-delivery vehicles, most of these studies were restricted to in vitro testing with few being tested in vivo [22-27]. Furthermore, only a limited number of nanocarriers has moved beyond the laboratory and has been tested in the clinic (see Table 2). The reasons for their inability to test several nanocarriers in the clinic are multifactorial and include inability to produce clinical grade nanocarriers in large quantities, inflammatory response [28-30], poor stability and short half-life of the nanocarrier in vivo [31,32], interaction with serum proteins and aggregation $[33,34]$, poor uptake of the nanocarrier by the tumors, and rapid clearance by macrophage and the reticuloendothelial system (RES) [35].

Methods to overcome some of these limitations included PEGylation of the nanocarriers using polyethylene glycol (PEG). Pegylated nanocarriers demonstrated improved stability in vivo, reduced RES clearance, and increased accumulation in tumors resulting in enhanced antitumor activity [36-38]. Similarly, studies using neutral or negatively charged nanocarriers have reported effective delivery of oligonucleotides, siRNA, and chemotherapeutic drugs [39, 40]. Despite the advances made with neutral and anionic lipid-based nanocarriers, they have not been developed and tested widely as tumor-suppressor gene-delivery vehicles for cancer therapy.

In 1998, Templeton et al. [41] reported that cationic DOTAP:cholesterol (DOTAP:Chol) lipid nanocarrier efficiently delivered plasmid DNA to the lung when administered intravenously. Findings by Gaensler et al. [42] concurred that DOTAP:Chol lipid nanocarrier to be an efficient gene-delivery vehicle. Crook et al. [43] reported that inclusion of cholesterol was important and a key to achieving stabilization of the DOTAP:Chol-nanocarrier and efficient gene transfer. The key feature that makes this nanocarrier better than previously tested lipid-based nanocarriers is its stability and reduced interaction with blood proteins in vivo 
TABLE 1: Tumor suppressor genes tested as cancer therapeutic in preclinical studies.

\begin{tabular}{|c|c|c|c|c|}
\hline TSG & Cancer & Animal model & Therapeutic outcomes & Molecular events \\
\hline E1A & Ovarian & $\begin{array}{l}\text { Intraperitoneal } \\
\text { tumor }\end{array}$ & $\begin{array}{c}\text { Reduced abdominal tumor } \\
\text { burden; increased animal } \\
\text { survival }\end{array}$ & $\begin{array}{c}\text { Apoptosis, reduced } \\
\text { ascites, and cell cycle } \\
\text { arrest }\end{array}$ \\
\hline p53 & Lung & $\begin{array}{l}\text { Subcutaneous } \\
\text { tumor; experimental } \\
\text { lung metastasis }\end{array}$ & $\begin{array}{l}\text { Tumor-growth inhibition; } \\
\text { reduced extrapulmonary } \\
\text { tumor nodules and increased } \\
\text { animal survival }\end{array}$ & $\begin{array}{l}\text { Cell cycle arrest, } \\
\text { apoptosis, } \\
\text { andantiangiogenesis }\end{array}$ \\
\hline Fhit & Lung & $\begin{array}{l}\text { Subcutaneous } \\
\text { tumor; experimental } \\
\text { lung metastasis }\end{array}$ & $\begin{array}{l}\text { Tumor-growth inhibition; } \\
\text { reduced extrapulmonary } \\
\text { tumor nodules and increased } \\
\text { animal survival }\end{array}$ & $\begin{array}{c}\text { Cell cycle arrest and } \\
\text { apoptosis }\end{array}$ \\
\hline IL-24 & Lung & $\begin{array}{l}\text { Subcutaneous } \\
\text { tumor; experimental } \\
\text { lung metastasis }\end{array}$ & $\begin{array}{l}\text { Tumor-growth inhibition; } \\
\text { reduced extrapulmonary } \\
\text { tumor nodules; increased } \\
\text { animal survival }\end{array}$ & $\begin{array}{c}\text { Cell cycle arrest, } \\
\text { apoptosis, } \\
\text { antiangiogenesis, and } \\
\text { autophagy proimmune } \\
\text { activity }\end{array}$ \\
\hline Fus1 & Lung & $\begin{array}{l}\text { Subcutaneous } \\
\text { tumor; experimental } \\
\text { lung metastasis }\end{array}$ & $\begin{array}{c}\text { Tumor-growth inhibition; } \\
\text { reduced extrapulmonary } \\
\text { tumor nodules; increased } \\
\text { animal survival }\end{array}$ & $\begin{array}{c}\text { Cell cycle arrest and } \\
\text { apoptosis }\end{array}$ \\
\hline BiKDD & Pancreas & $\begin{array}{l}\text { Subcutaneous } \\
\text { tumor; orthotopic } \\
\text { tumor }\end{array}$ & $\begin{array}{l}\text { Tumor-growth inhibition; } \\
\text { reduced metastasis, increased } \\
\text { animal survival }\end{array}$ & Apoptosis \\
\hline
\end{tabular}

which is contributed by the inclusion of cholesterol [41]. Another key feature that likely contributes to its effectiveness is that the lipid-nanocarrier, when mixed with DNA, forms unique bilamellar vase-like structures that keep the DNA intact from rapid degradation [41]. However, it is likely that additional factors that are unknown at the present time may contribute to its effectiveness.

Based on these reports, we initiated preclinical studies in our laboratory and tested whether DOTAP:Chol-lipid nanocarrier could efficiently deliver tumor suppressor genes when administered systemically and control metastatic lung tumors. Size fractionation studies showed our lipid nanocarrier was $200-400 \mathrm{~nm}$ in size and had a positive charge of $40 \pm 2 \mathrm{mV}[44,45]$. The nanocarriers are stable $+4^{\circ} \mathrm{C}$ for over a period of one month when stored as empty nanocarriers and for at least $48 \mathrm{~h}$ when mixed with DNA. Although one may argue that our nanocarriers are large, results from our studies, as discussed below, support particle size of 200$400 \mathrm{~nm}$ to be optimal and to strike a balance between tumor uptake and macrophage clearance. Furthermore, we believe that the size of the nanocarrier will need to be varied and optimized depending on the disease to be treated and that the concept of one-size-fits-all disease treatments cannot be applied.

In vitro studies showed transfection efficiency mediated by the nanocarrier varied among cell types that correlated with transgene expression [44, 46, 47]. Transgene expression was observed to be detectable as early as $12 \mathrm{~h}$ after transfection and was detectable up to $72 \mathrm{~h}$ after transfection albeit expression levels decreased over time. The transfection efficiency and transgene expression were observed to be consistent in a given cell line even when different tumor suppressor genes or marker genes were used. One factor that affected transfection efficiency and transgene expression was the size of plasmid contained in the nanocarrier. In general, a nanocarrier containing a plasmid that was $3-4 \mathrm{~Kb}$ in size produced higher transfection compared to nanocarrier containing a plasmid that was greater than $4 \mathrm{~Kb}$ in size. Furthermore, the DNA-containing nanocarrier was stable for at least $48 \mathrm{~h}$ when stored at $+4^{\circ} \mathrm{C}$ and produced comparable transfection efficiency and transgene expression in tumor cells when compared to that produced by cells treated with a freshly prepared DNA-containing nanocarrier.

In vivo studies were initially focused on biodistribution and toxicity of the DNA-containing nanocarrier in immunocompetent mice. Biodistribution studies showed that the DNA-nanocarrier primarily localized to the lung when injected intravenously. However, over time the nanocarrier exited the lung and was detectable in other organs (liver, spleen, kidney etc). Toxicity studies demonstrated a dosedependent response with LD10 being in the range of 55$70 \mu \mathrm{g}$ of DNA in the lipid nanocarrier and depended on the backbone of the plasmid DNA. The therapeutic gene contributed very little to toxicity (unpublished data).

We next investigated the therapeutic effects of a TSGcontaining nanocarrier on human lung tumor xenografts established in nude mice. Marker gene expression showed marked transgene expression when injected intratumorally into subcutaneous lung tumor xenografts [40]. Efficacy studies showed that a TSGs-containing (p53, Fhit, Fus1, TSG101, or IL-24) nanocarrier, when administered intratumorally, produced significant growth inhibition compared to tumor growth inhibition produced by nanocarrier treatment containing control plasmid DNA [44, 46, 47]. Growth inhibition, 
TABLE 2: Synthetic nanocarriers tested for cancer gene therapy in human Phase I clinical trials.

\begin{tabular}{|c|c|c|c|}
\hline Nanocarrier & $\begin{array}{c}\text { Therapeutic } \\
\text { gene }\end{array}$ & Cancer & Route of administration \\
\hline $\begin{array}{l}\text { DC (3 beta- }\left[\mathrm{n}-\left(\mathrm{N}^{\prime}, \mathrm{N}^{\prime} \text {-dimethylaminoethane)- }\right.\right. \\
\text { carbamoyl] cholesterol }) \text { : DOPE } \\
\text { (dioleoylphosphatidylethanolamine) }\end{array}$ & E1A & Breast/ovarian & Intratumoral (it)/intraperitoneal (ip) \\
\hline $\begin{array}{l}\text { DC ( } 3 \text { beta- }\left[\mathrm{n}-\left(\mathrm{N}^{\prime}, \mathrm{N}^{\prime} \text {-dimethylaminoethane)- }\right.\right. \\
\text { carbamoyl }] \text { cholesterol }) \text { : Chol } \\
\text { (cholesterol) }\end{array}$ & EGFR & Head \& neck & Intratumoral \\
\hline $\begin{array}{l}\text { DOTAP (N-[1-(2, 3-dioleoyloxy)propyl]- } \\
\text { N,N,N-trimethylammonium Chloride): DOPE } \\
\text { (dioleoylphosphatidylethanolamine) }\end{array}$ & p53 & Solid tumor & Intravenous (iv) \\
\hline $\begin{array}{l}\text { DOTAP (N-[1-(2, 3-dioleoyloxy)propyl]- } \\
\text { N,N,N-trimethylammonium Chloride): Chol } \\
\text { (cholesterol) }\end{array}$ & BiKDD & $\begin{array}{l}\text { Pancreatic } \\
\text { cancer }\end{array}$ & Intravenous (iv) \\
\hline $\begin{array}{l}\text { DOTAP (N-[1-(2, 3-dioleoyloxy)propyl]- } \\
\text { N,N,N-trimethylammonium Chloride): Chol } \\
\text { (cholesterol) }\end{array}$ & E1A & Breast/ovarian & Intravenous (iv) \\
\hline $\begin{array}{l}\text { DOTAP (N-[1-(2, 3-dioleoyloxy)propyl]- } \\
\text { N,N,N-trimethylammonium Chloride): Chol } \\
\text { (cholesterol) }\end{array}$ & Fus1 & Lung & Intravenous (iv) \\
\hline $\begin{array}{l}\text { DOTMA (N-[1-(2,3-dioleyloxy)propyl]- } \\
\text { N,N,N-trimethylammonium Chloride): Chol } \\
\text { (cholesterol) }\end{array}$ & IL-2 & Head \& neck & Intratumoral (it) \\
\hline
\end{tabular}

Source: www.cancertrials.gov.

produced by TSG-containing nanocarrier was independent of the tumor model, as comparable growth-inhibitory effects were observed in human H1299 lung tumor and murine UV2237 tumor xenografts established in nude mice and $\mathrm{C} 3 \mathrm{H}$ mice, respectively [46]. Furthermore, repeated treatments showed greater tumor-growth inhibition that correlated with increased transgene expression when compared to growthinhibitory effects produced by single treatments. Our study also showed that the therapeutic effect produced by p53 TSGcontaining nanocarrier treatment was independent of the endogenous p53 status of the treated tumor. Additionally, the therapeutic effect produced using various TSGs was comparable, albeit differences existed among tumor types. These results provide evidence and support intratumoral treatments of localized tumors such as cancer of the head and neck that are unresectable with TSG-containing nanocarrier. It is envisioned that such localized intratumoral treatments with TSG-containing nanocarrier will reduce the tumor burden and make the tumor accessible to surgery and radiation therapy.

Since our objective and goal was to test the nanocarrier as a systemic gene-delivery vehicle for treatment of metastatic disease, we conducted in vivo studies using experimental tumor-metastasis models. Human H1299 (p53 null) and A549 (p53 wild-type) tumor cells were injected intravenously via tail vein to establish experimental lung metastasis in SCID/Beige and nude mice, respectively. Mice received daily intravenous treatments with a p53 TSG-containing nanocarrier for a total of six doses. At four weeks after the last treatment mice were euthanized, lungs were harvested and examined for the number of pulmonary nodules.
A significant reduction in the number of pulmonary tumor nodules were observed in mice receiving p53 TSG nanocarrier treatment compared to the number of pulmonary tumor nodules in mice receiving control DNA-containing nanocarrier treatment [44]. Histopathological examination of the lungs from mice receiving p53 TSG-containing nanocarrier treatment showed few tumors with evidence of tumor cells undergoing apoptotic cell death compared to the number of tumors in the lungs of control mice and very few tumor cells undergoing apoptosis.

Since the six-day treatment with p53 TSG-containing nanocarrier did not completely abolish pulmonary tumor growth we next determined whether these tumors will regrow and if they can be treated with a second cycle of treatment akin to that practiced in the clinic. For this purpose, mice bearing experimental lung tumors were divided into two groups. One group of mice $(n=8)$ received the initial six treatments with p53 TSG nanocarrier (day 1$6)$. A second group of mice $(n=8)$ received the initial six treatments with p53 TSG nanocarrier (day 1-6) and a second cycle of six treatments starting on day 30 (day 30-35). Mice $(n=4)$ from each group were euthanized on day 28 and on day 42. Lungs were harvested from the euthanized mice and the number of lung tumor nodules counted. Our results showed that a greater reduction in the number of pulmonary tumor nodules in the lungs of mice receiving two cycles of p53 TSG nanocarrier treatment compared to the reduction in tumor nodules in the lungs of mice receiving single cycle of p53 TSG nanocarrier treatment (unpublished data). Our results demonstrate repeated cycles of treatment are feasible and that they produced a greater therapeutic effect. 
We next determined the therapeutic effects of p53 TSGcontaining nanocarrier in disseminated tumor mouse model. Injection of H1299 lung tumor cells into SCID/Beige mice results in disseminated tumors in various organs [44]. Treating these mice with the p53 TSG nanocarrier intravenously resulted in prolonged animal survival compared to survival of control mice that were either untreated or treated with a control plasmid DNA-containing nanocarrier [44].

Effective gene-delivery mediated by the nanocarrier was not restricted to p53 TSG therapy or to lung tumor models. Delivery of Fhit and Fus1 TSGs, that are frequently lost in human lung cancer, produced therapeutic effects that were similar to the therapeutic effects observed with p53 [44, 47]. Furthermore, combination of Fus1-containing nanocarrier with chemotherapy was shown to produce additive to synergistic therapeutic effect [48]. Similarly, systemic therapy with IL-24-containing nanocarrier inhibited human lung tumor and murine fibrosarcoma growth established in nude mice and immunocompetent $\mathrm{C} 3 \mathrm{H}$ mice, respectively [46]. In all of these studies repeated treatments resulted in additive increases in transgene expression in the tumors with minimal expression in normal tissues adjacent to the tumor [49], a finding that was in contrast to the report by $\mathrm{Li}$ et al. [50] who showed repeated treatments reduced transgene expression due to induction of treatment-related inflammatory response. The differences in the outcomes were due to difference in the animal models used. We demonstrated that mice bearing tumors produced immunosuppressive factors within the tumor microenvironment that altered the host immune pathology resulting in no inhibitory effects on transgene expression [51]. Additionally, nanocarrier tracking studies demonstrated tumors that were larger in size had more nanocarriers compared to tumors that were smaller in size [49]. The uptake of the nanocarriers involved tumor-mediated phagocytosis. Furthermore, the inflammatory response produced in the tumor-bearing mice was markedly reduced. On the contrary, if the mice did not bear any tumors then the nanocarrier was widely distributed in the lung, and induction of treatment-related inflammatory response and shutting down of transgene expression following repeated treatments was observed [44, 49, 52]. Thus, the outcomes of repeated nanocarrier treatment and transgene expression can be regulated by the host pathology and disease conditions and therefore need to be considered during drug development.

More recently, we have tested the systemic therapeutic effects of IL-24-containing nanocarrier treatments in a metastatic melanoma model. Nude mice injected with human melanoma (MeWo) tumor cells that are genetically modified to express the green fluorescent protein (GFP) produced tumors that metastasized to the lung, liver, brain, and several other organs and visible under bright light and fluorescent light (Figure 1). Mice injected with MeWoGFP cells and bearing experimental metastasis were divided into the following groups: no treatment; treatment with IL-24 plasmid DNA; treatment with empty nanocarrier; treatment with IL-24-containing nanocarrier. Mice were treated twice a week (50 $\mu \mathrm{g}$ DNA) until the study was terminated. Treatment of these mice having experimental metastasis intravenously with IL-24-nanocarriers resulted in prolonged animal survival compared to survival of mice that received other treatments or no treatment (Figure 2). These studies showed systemic treatment with our nanocarrier delivers therapeutic genes and produces effective anticancer activity.

We next determined whether our nanocarrier can deliver TSGs to ovarian cancer when administered intraperitoneally (i.p.) and whether it was superior to adenovirus-mediated gene-delivery in producing a therapeutic effect. Nude mice were injected into the peritoneum with human ovarian MDAH2774 tumor cells. The mice rapidly form ascites with disease progression and at which time if untreated they will have to be euthanized. These i.p. tumor-bearing mice were divided into groups and treated as follows: treated with IL-24-containing nanocarrier, treated with adenovirus (Ad)-IL-24, treated with Ad-luciferase (Luc), or treated with phosphate buffered saline (PBS). Animals were monitored daily and animal survival recorded. As shown in Figure 3, mice receiving IL-24-containing nanocarrier showed a trend for increased survival compared to all other treatment groups. Our preliminary results showed nanocarrier-based therapy was more effective than adenovirus-based therapy in controlling tumor growth and progression for ovarian cancer. Finally, our studies demonstrate DOTAP:Chol-based nanocarrier is efficient in delivering therapeutics genes to local and metastatic tumor sites and can be administered via various routes resulting in enhanced therapeutic effects in preclinical models.

2.2. Clinical Studies. On the basis of our preclinical studies, a Phase I clinical trial for the systemic treatment of nonsmall cell lung cancer (NSCLC) has been initiated at the University of Texas MD Anderson Cancer Center, Houston, Texas, USA [53]. This trial which is a first of its kind aims at testing whether DOTAP:Chol. nanocarrier-containing a TSG, Fus1, can be administered intravenously in patients with recurrent/metastatic lung cancer previously treated with platinum-based chemotherapy. Fus1 is a TSG located on chromosome 3p21.3 $[54,55]$. The rationale for selecting Fus1 for NSCLC therapy is because it is frequently lost or deleted in more than $60 \%$ of patients diagnosed with lung cancer [56]. Additionally, studies have shown that Fus1 effectively suppressed lung-tumor growth in vivo when used as monotherapy or in combination with other drugs [57-59]. The primary objective of this trial is to treat patients with an escalating dose $(0.01-0.09 \mathrm{mg} / \mathrm{Kg})$ of Fus1-containing nanocarrier at a three-week interval and determine the maximum tolerated dose (MTD). Up to date, 23 patients have been entered on the study trial and have received one or more Fus1-containing nanocarrier treatment. Preliminary results demonstrate Fus1 nanocarrier treatment is well tolerated with no major treatment-related toxicity [53]. Furthermore, MTD is yet to be determined, and the trial is open and continuing to accrue patients. The final results of the Fus1 nanocarrier treatment is expected to be available upon completion of the trial. The outcome of this trial will facilitate the design of future TSG-nanocarrier-based Phase I/II clinical trials for lung cancer. 

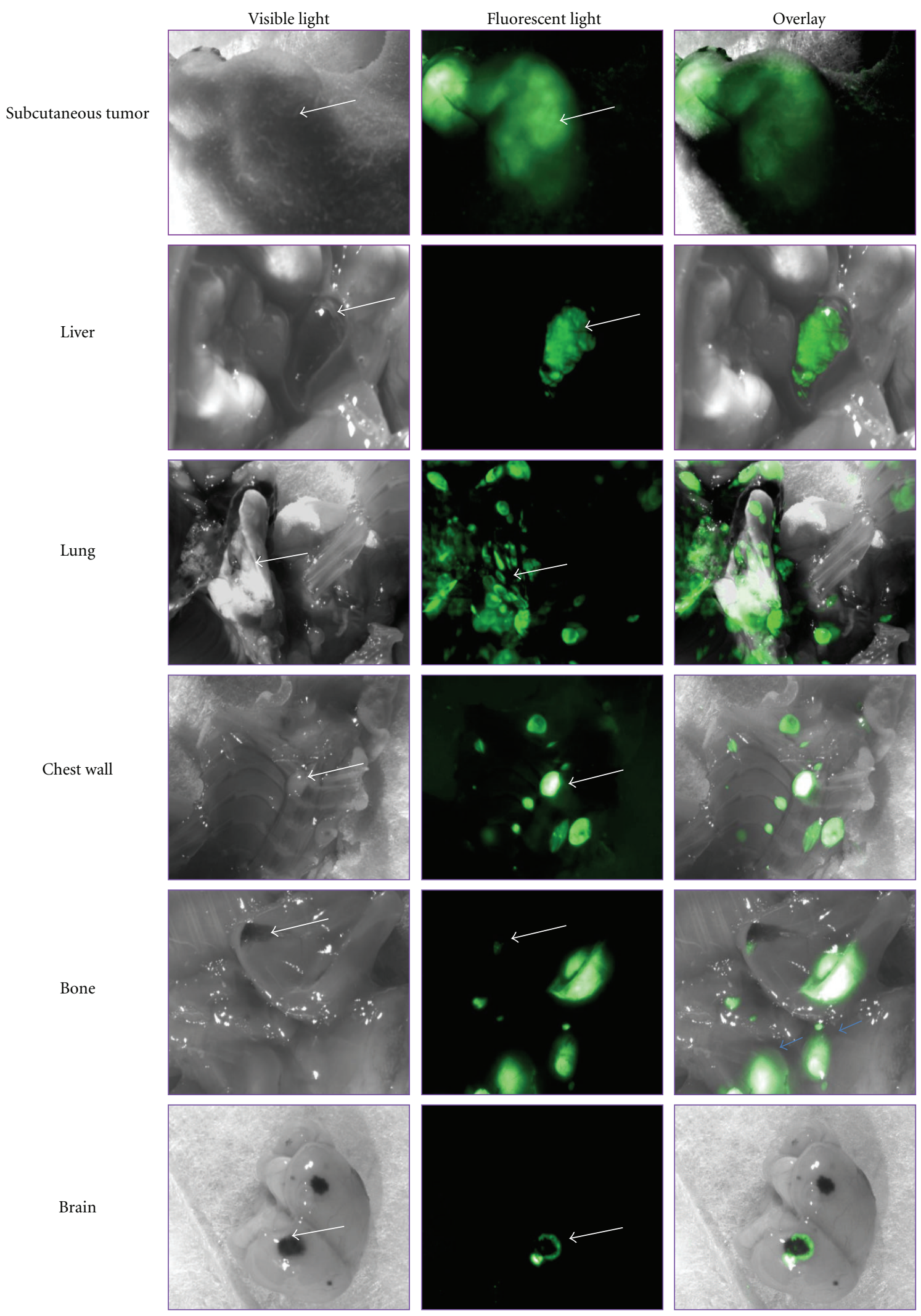

FIGURE 1: Detection of melanoma metastasis by fluorescent imaging. Human melanoma MeWo cell line was stably transfected and selected for GFP positive clones. The MeWo-GFP cells $\left(5 \times 10^{6}\right)$ were injected intravenously via tail vein in nude mice. Mice were euthanized after four to five weeks and observed under normal white light and under fluorescent light. Melanoma tumors were detectable under white light in various organs. However, an increased number of GFP-positive tumors (green fluorescence) were observed under fluorescence light in various organs indicating melanoma metastasis. Additionally, tumors not visible under white light were detected by fluorescence. 


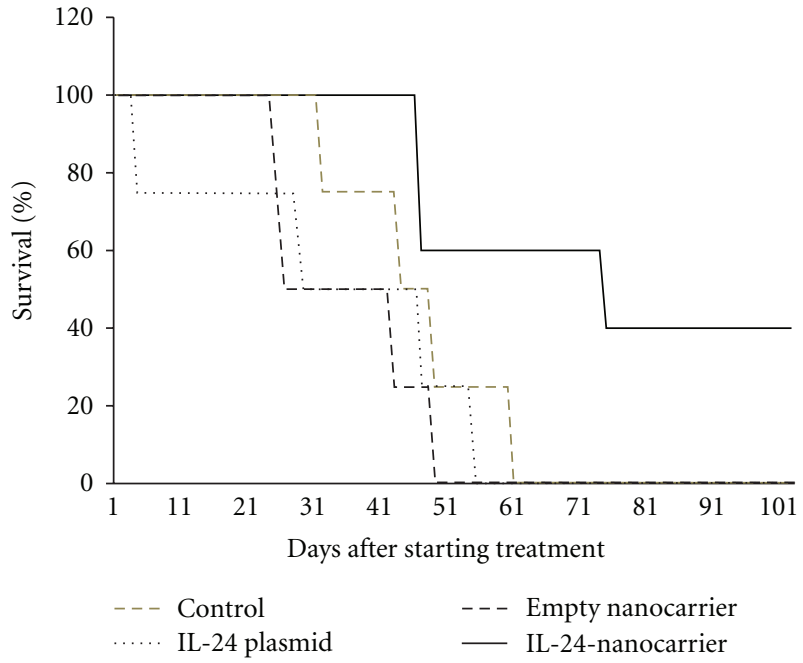

Figure 2: IL-24 nanotherapy improves animal survival. Nude mice were injected with MeWo-GFP. Ten days after tumor cell injection mice were divided into four groups: group received no treatment; group 2 received IL-24 plasmid DNA; group 3 received empty nanocarrier; group 4 received IL-24-containing nanocarrier $(50 \mu \mathrm{g}$ DNA). Treatment was twice a week and administered intravenously for six weeks. Mice were monitored for animal survival. Mice receiving IL-24-containing nanocarrier therapy showed increased survival compared to all other treatment groups.

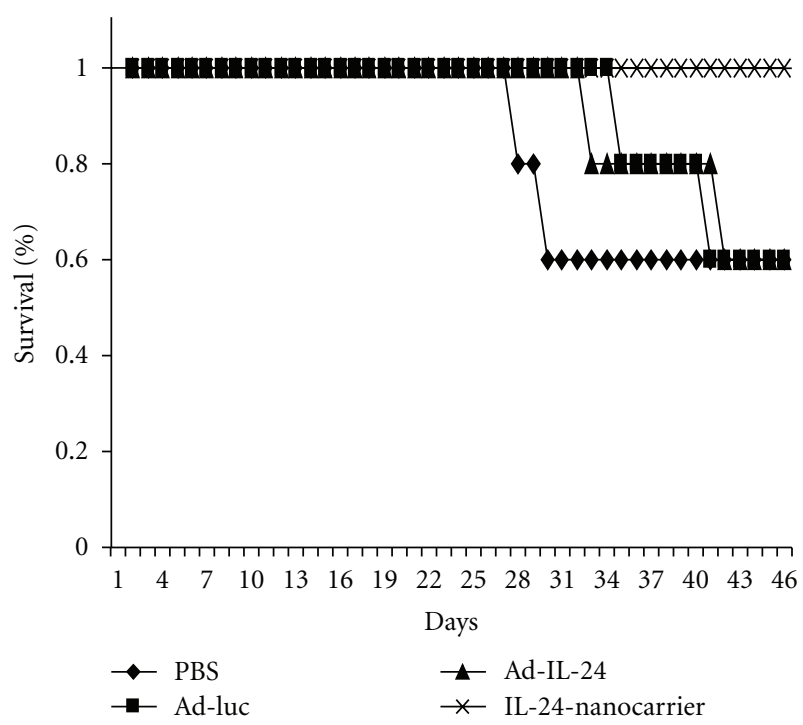

Figure 3: IL-24 nanotherapy for ovarian cancer. MDAH2774 $(1 \times$ $10^{6}$ ) cells were injected into the peritoneal cavity of nude mice. Mice were then divided into groups and treated with IL-24-containing nanocarrier intraperitoneally (i.p.). Mice that were treated with phosphate buffered saline (PBS), treated with adenovirus (Ad)IL-24, or treated with Ad-luciferase (Luc) served as controls. An increase in animal survival was observed in mice that received IL24-containing nanotherapy compared to all other treatment groups.

On the basis of our preclinical studies and the Fus1containing nanocarrier Phase I clinical trial, two additional
Phase I clinical trials for the treatment of pancreatic cancer, ovarian, and breast cancer have been approved by the Food and Drug Administration (FDA) (see Table 2). These trials will be conducted at the MD Anderson Cancer Center, Houston, TX, USA. Both of these trials have objectives and endpoints similar to the Fus1 trial. The only difference is the therapeutic gene to be used for these cancer types is different and not Fus1. For pancreatic cancer, a proapoptotic gene called Bcl-2 interacting killer (Bik) gene (BikDD) will be used for therapy [60]. The uniqueness is that BikDD gene expression will be under the control of cholecystokinin type A receptor (CCKAR) that will be conditionally regulated by VP16-GAL4-WPRE integrated systemic amplifier (VISA). This system is tumor selective and high BikDD protein expression is expected to occur in cancer cells with minimal protein expression occurring in surrounding normal tissues, and thus eliminating unwanted cytotoxicity. The objective of the pancreatic cancer Phase I clinical trial is to determine the MTD and optimal biological active dose (OBAD) compared with the clinical response. The trial has not been completed, and the results from this trial are therefore pending.

In the Phase I clinical trial planned for breast cancer treatment, the therapeutic gene to be incorporated into the DOTAP:Chol. nanocarrier is the E1A tumor suppressor gene. E1A gene introduction into breast cancer cells induces cell cycle arrest and cell death both in vitro and in vivo [61]. Additionally, E1A has previously been tested in a Phase I clinical trial for treatment of breast and ovarian cancer patients. Although results from the early trial did not show any therapeutic benefits, it demonstrated E1A treatment was safe [62]. This trial, like the pancreatic trial, is currently open for patient accrual and not completed. Therefore, results from this trial will remain unknown for, at least, the next one to two years.

More recently plans for a Phase I clinical trial testing systemic IL-24 nanocarrier therapy for metastatic melanoma is underway. Preclinical efficacy and toxicity studies, that are prerequisites for submitting IL-24 nanocarrier as investigational new drug (IND), have been completed. The IL-24 nanocarrier phase I clinical trial is yet to receive approval from the Food and Drug Administration (FDA) and will be conducted at the MD Anderson Cancer Center, Houston, TX, USA.

It is evident from the number of Phase I clinical trials that have been initiated on the basis of our laboratory findings that the lipid-based DOTAP:Chol nanocarrier is useful for systemic delivery of cancer gene therapeutics. Successful translation of laboratory research to a clinic such as ours described above will lead to promising cancer treatment strategies and therapies. It is anticipated that additional laboratory research will be translated to the clinic in the next few years.

\section{Conclusions}

Since the inception and testing of gene-based therapies for cancer in the early 1990s, significant progress in the understanding of the biology of the disease and vector development has been made. Failure to control and/or 
eradicate cancer using virus-based cancer gene therapy has led to advancement of the nonviral delivery field. Despite skepticism and unexpected gene therapy related deaths, progress has been made in the area of cancer gene therapy and will continue to be made. It is evident from the progress made in our own laboratory, by moving laboratory research to the clinic one could successfully translate future research for cancer therapy. Since combination therapies have often been reported to produce additive to synergistic therapeutic effect, it is not far from testing Fus1 nanocarrier in combination with conventional chemotherapies or molecularly targeted therapies. For example one could combine Fus1 nanocarrier with the epidermal growth factor receptor (EGFR) kinase-targeted inhibitors for treatment of lung cancer. Similarly, IL-24 nanocarrier therapy can be combined with Raf-targeted inhibitor (sorafenib) or alternatively with the chemotherapeutic Temozolomide for treatment of metastatic melanoma. Given the possibility of testing various combinations, it is critical that the ongoing Phase I clinical trials are successful so that future clinical trials incorporating combination therapies can be designed and tested.

With the advent of nanotechnology and its application to cancer medicine, novel nonviral vector-based nanocarriers that are multifunctional in their properties have been developed and are currently being tested in several laboratories around the world $[24,63-65]$. It is thus not far from the near future that several Phase I clinical trials based on novel nanoformulations and properties will be initiated for testing drugs, genes, siRNA, aptamers, or molecular imaging agents for cancer diagnosis and therapy $[27,66,67]$.

\section{Acknowledgments}

The authors thank all the laboratory members who have contributed to various aspects of the nanotherapy project. Grant funding received from the National Institutes of Health (NIH) R01 CA113450 and a Career Development Grant received from the Melanoma SPORE P50 CA093459 are acknowledged.

\section{References}

[1] American Cancer Society, Cancer Facts \& Figures 2009, American Cancer Society, Atlanta, Ga, USA, 2009.

[2] R. T. Greenlee, T. Murray, S. Bolden, and P. A. Wingo, "Cancer statistics, 2000," Ca: A Cancer Journal for Clinicians, vol. 50, no. 1, pp. 7-33, 2000.

[3] A. Mhashilkar, S. Chada, J. A. Roth, and R. Ramesh, "Gene therapy: therapeutic approaches and implications," Biotechnology Advances, vol. 19, no. 4, pp. 279-297, 2001.

[4] C. J. Sherr, "Principles of tumor suppression," Cell, vol. 116, no. 2, pp. 235-246, 2004.

[5] A. J. Levine and M. Oren, "The first 30 years of p53: growing ever more complex," Nature Reviews Cancer, vol. 9, no. 10, pp. 749-758, 2009.

[6] D. Lohmann, "Retinoblastoma," Advances in Experimental Medicine and Biology, vol. 685, pp. 220-227, 2010.

[7] J. A. Roth, D. Nguyen, D. D. Lawrence et al., "Retrovirusmediated wild-type p53 gene transfer to tumors of patients with lung cancer," Nature Medicine, vol. 2, no. 9, pp. 985-991, 1996.

[8] S. G. Swisher, J. A. Roth, J. Nemunaitis et al., "Adenovirusmediated p53 gene transfer in advanced non-small-cell lung cancer," Journal of the National Cancer Institute, vol. 91, no. 9, pp. 763-771, 1999.

[9] J. Nemunaitis, S. G. Swisher, T. Timmons et al., "Adenovirusmediated p53 gene transfer in sequence with cisplatin to tumors of patients with non-small-cell lung cancer," Journal of Clinical Oncology, vol. 18, no. 3, pp. 609-622, 2000.

[10] S. W. Zhang, S. W. Xiao, C. Q. Liu et al., "Treatment of head and neck squamous cell carcinoma by recombinant adenovirus-p53 combined with radiotherapy: a phase II clinical trial of 42 cases," Zhonghua Yi Xue Za Zhi, vol. 83, no. 23, pp. 2023-2028, 2003.

[11] E. Marshall, "Gene therapy death prompts review of adenovirus vector," Science, vol. 286, no. 5448, pp. 2244-2245, 1999.

[12] T. Kafri, D. Morgan, T. Krahl, N. Sarvetnick, L. Sherman, and I. Verma, "Cellular immune response to adenoviral vector infected cells does not require de novo viral gene expression: implications for gene therapy," Proceedings of the National Academy of Sciences of the United States of America, vol. 95, no. 19, pp. 11377-11382, 1998.

[13] Y. Yang, H. C. J. Ertl, and J. M. Wilson, "MHC class I-restricted cytotoxic T lymphocytes to viral antigens destroy hepatocytes in mice infected with E1-deleted recombinant adenoviruses," Immunity, vol. 1, no. 5, pp. 433-442, 1994.

[14] Y. Yang, J. Ku, Q. Su, H. C. J. Ertl, and J. M. Wilson, "Immune responses to viral antigens versus transgene product in the elimination of recombinant adenovirus-infected hepatocytes in vivo," Gene Therapy, vol. 3, no. 2, pp. 137-144, 1996.

[15] T. Niidome and L. Huang, "Gene therapy progress and prospects: nonviral vectors," Gene Therapy, vol. 9, no. 24, pp. 1647-1652, 2002.

[16] S. D. Li and L. Huang, "Gene therapy progress and prospects: non-viral gene therapy by systemic delivery," Gene Therapy, vol. 13, no. 18, pp. 1313-1319, 2006.

[17] C. Huisman, E. F. Smit, G. Giaccone, and P. E. Postmus, "Second-line chemotherapy in relapsing or refractory nonsmall-cell lung cancer: a review," Journal of Clinical Oncology, vol. 18, no. 21, pp. 3722-3730, 2000.

[18] Y. A. Hannun, "Apoptosis and the dilemma of cancer chemotherapy," Blood, vol. 89, no. 6, pp. 1845-1853, 1997.

[19] M. V. Yezhelyev, X. Gao, Y. Xing, A. Al-Hajj, S. Nie, and R. M. O'Regan, "Emerging use of nanoparticles in diagnosis and treatment of breast cancer," The Lancet Oncology, vol. 7, no. 8, pp. 657-667, 2006.

[20] L. Xu, C. C. Huang, W. Huang et al., "Systemic tumortargeted gene delivery by anti-transferrin receptor $\mathrm{scFv}$ immunoliposomes," Molecular Cancer Therapeutics, vol. 1, no. 5, pp. 337-346, 2002.

[21] S. Sundaram, R. Trivedi, C. Durairaj, R. Ramesh, B. K. Ambati, and U. B. Kompella, "Targeted drug and gene delivery systems for lung cancer therapy," Clinical Cancer Research, vol. 15, no. 23, pp. 7299-7308, 2009.

[22] C. Srinivasan and D. J. Burgess, "Optimization and characterization of anionic lipoplexes for gene delivery," Journal of Controlled Release, vol. 136, no. 1, pp. 62-70, 2009.

[23] P. Sun, M. Zhong, X. Shi, and Z. Li, "Anionic LPD complexes for gene delivery to macrophage: preparation, characterization and transfection in vitro," Journal of Drug Targeting, vol. 16, no. 9, pp. 668-678, 2008. 
[24] A. M. Smith, S. Dave, S. Nie, L. True, and X. Gao, "Multicolor quantum dots for molecular diagnostics of cancer," Expert Review of Molecular Diagnostics, vol. 6, no. 2, pp. 231-244, 2006.

[25] H. Devalapally, D. Shenoy, S. Little, R. Langer, and M. Amiji, "Poly(ethylene oxide)-modified poly(beta-amino ester) nanoparticles as a $\mathrm{pH}$-sensitive system for tumor-targeted delivery of hydrophobic drugs: part 3. Therapeutic efficacy and safety studies in ovarian cancer xenograft model," Cancer Chemotherapy and Pharmacology, vol. 59, no. 4, pp. 477-484, 2007.

[26] L. H. Reddy, K. Vivek, N. Bakshi, and R. S. R. Murthy, "Tamoxifen citrate loaded solid lipid nanoparticles $\left(\mathrm{SLN}^{\mathrm{TM}}\right)$ : preparation, characterization, in vitro drug release, and pharmacokinetic evaluation," Pharmaceutical Development and Technology, vol. 11, no. 2, pp. 167-177, 2006.

[27] O. C. Farokhzad, J. M. Karp, and R. Langer, "Nanoparticleaptamer bioconjugates for cancer targeting," Expert Opinion on Drug Delivery, vol. 3, no. 3, pp. 311-324, 2006.

[28] Y. Liu, L. C. Mounkes, H. D. Liggitt et al., "Factors influencing the efficiency of cationic liposome-mediated intravenous gene delivery," Nature Biotechnology, vol. 15, no. 2, pp. 167-173, 1997.

[29] N. S. Yew, K. X. Wang, M. Przybylska et al., "Contribution of plasmid DNA to inflammation in the lung after administration of cationic lipid:pDNA complexes," Human Gene Therapy, vol. 10, no. 2, pp. 223-234, 1999.

[30] B. D. Freimark, H. P. Blezinger, V. J. Florack et al., "Cationic lipids enhance cytokine and cell influx levels in the lung following administration of plasmid: cationic lipid complexes," Journal of Immunology, vol. 160, no. 9, pp. 4580-4586, 1998.

[31] A. Pathak, S. Patnaik, and K. C. Gupta, "Recent trends in nonviral vector-mediated gene delivery," Biotechnology Journal, vol. 4, no. 11, pp. 1559-1572, 2009.

[32] M. Ramezani, M. Khoshhamdam, A. Dehshahri, and B. Malaekeh-Nikouei, "The influence of size, lipid composition and bilayer fluidity of cationic liposomes on the transfection efficiency of nanolipoplexes," Colloids and Surfaces B, vol. 72, no. 1, pp. 1-5, 2009.

[33] J. P. Yang and L. Huang, "Overcoming the inhibitory effect of serum on lipofection by increasing the charge ratio of cationic liposome to DNA," Gene Therapy, vol. 4, no. 9, pp. 950-960, 1997.

[34] S. Li, W. C. Tseng, D. Beer Stolz, S. P. Wu, S. C. Watkins, and L. Huang, "Dynamic changes in the characteristics of cationic lipidic vectors after exposure to mouse serum: implications for intravenous lipofection," Gene Therapy, vol. 6, no. 4, pp. 585594, 1999.

[35] W. Li and F. C. Szoka, "Lipid-based nanoparticles for nucleic acid delivery," Pharmaceutical Research, vol. 24, no. 3, pp. 438 449, 2007.

[36] V. P. Torchilin, "Polymer-coated long-circulating microparticulate pharmaceuticals," Journal of Microencapsulation, vol. 15, no. 1, pp. 1-19, 1998.

[37] F. Szoka and D. Papahadjopoulos, "Comparative properties and methods of preparation of lipid vesicles (liposomes)," Annual Review of Biophysics and Bioengineering, vol. 9, pp. 467-508, 1980.

[38] K. Iga, K. Ohkouchi, Y. Ogawa, and H. Toguchi, "Membrane modification by negatively charged stearyl-polyoxyethylene derivatives for thermosensitive liposomes: reduced liposomal aggregation and avoidance of reticuloendothelial system uptake," Journal of Drug Targeting, vol. 2, no. 3, pp. 259-267, 1994.
[39] M. M. Mady, M. M. Ghannam, W. A. Khalil et al., "Efficient gene delivery with serum into human cancer cells using targeted anionic liposomes," Journal of Drug Targeting, vol. 12, no. 1, pp. 11-18, 2004.

[40] E. Fattal, P. Couvreur, and C. Dubernet, "”Smart” delivery of antisense oligonucleotides by anionic $\mathrm{pH}$-sensitive liposomes," Advanced Drug Delivery Reviews, vol. 56, no. 7, pp. 931-946, 2004.

[41] N. S. Templeton, D. D. Lasic, P. M. Frederik, H. H. Strey, D. D. Roberts, and G. N. Pavlakis, "Improved DNA: liposome complexes for increased systemic delivery and gene expression," Nature Biotechnology, vol. 15, no. 7, pp. 647-652, 1997.

[42] K. M. L. Gaensler, G. Tu, S. Bruch et al., "Fetal gene transfer by transuterine injection of cationic liposome-DNA complexes," Nature Biotechnology, vol. 17, no. 12, pp. 1188-1192, 1999.

[43] K. Crook, B. J. Stevenson, M. Dubouchet, and D. J. Porteous, "Inclusion of cholesterol in DOTAP transfection complexes increases the delivery of DNA to cells in vitro in the presence of serum," Gene Therapy, vol. 5, no. 1, pp. 137-143, 1998.

[44] R. Ramesh, T. Saeki, N. S. Templeton et al., "Successful treatment of primary and disseminated human lung cancers by systemic delivery of tumor suppressor genes using an improved liposome vector," Molecular Therapy, vol. 3, no. 3, pp. 337-350, 2001.

[45] B. Gopalan, I. Ito, C. D. Branch, C. Stephens, J. A. Roth, and R. Ramesh, "Nanoparticle based systemic gene therapy for lung cancer: molecular mechanisms and strategies to suppress nanoparticle-mediated inflammatory response," Technology in Cancer Research and Treatment, vol. 3, no. 6, pp. 647-657, 2004.

[46] R. Ramesh, I. Ito, Y. Saito et al., "Local and systemic inhibition of lung tumor growth after nanoparticle-mediated mda-7/IL24 gene delivery," DNA and Cell Biology, vol. 23, no. 12, pp. 850-857, 2004.

[47] I. Ito, L. Ji, F. Tanaka et al., "Liposomal vector mediated delivery of the $3 p$ FUS1 gene demonstrates potent antitumor activity against human lung cancer in vivo," Cancer Gene Therapy, vol. 11, no. 11, pp. 733-739, 2004.

[48] W. G. Deng, G. Wu, K. Ueda, K. Xu, J. A. Roth, and L. Ji, "Enhancement of antitumor activity of cisplatin in human lung cancer cells by tumor suppressor FUS1," Cancer Gene Therapy, vol. 15, no. 1, pp. 29-39, 2008.

[49] I. Ito, G. Began, I. Mohiuddin et al., "Increased uptake of liposomal-DNA complexes by lung metastases following intravenous administration," Molecular Therapy, vol. 7, no. 3, pp. 409-418, 2003.

[50] S. Li, S. P. Wu, M. Whitmore et al., "Effect of immune response on gene transfer to the lung via systemic administration of cationic lipidic vectors," American Journal of Physiology, vol. 276, no. 5, pp. L796-L804, 1999.

[51] I. Ito, T. Saeki, I. Mohuiddin et al., "Persistent transgene expression following intravenous administration of a liposomal complex: role of interleukin-10-mediated immune suppression," Molecular Therapy, vol. 9, no. 3, pp. 318-327, 2004.

[52] R. Ramesh, "Nanoparticle-mediated gene delivery to the lung," Methods in Molecular Biology, vol. 434, pp. 301-331, 2008.

[53] C. Lu, D. J. Stewart, L. Ji et al., "A phase I trial of intravenous therapy with tumor suppressor Fus1-nanoparticles for recurrent/metastatic lung cancer," Journal of Clinical Oncology, vol. 27, abstract e19065, 2009.

[54] M. I. Lerman and J. D. Minna, “The 630-kb lung cancer homozygous deletion region on human chromosome 3 p21.3: 
identification and evaluation of the resident candidate tumor suppressor genes," Cancer Research, vol. 60, no. 21, pp. 61166133, 2000.

[55] E. R. Zabarovsky, M. I. Lerman, and J. D. Minna, "Tumor suppressor genes on chromosome $3 p$ involved in the pathogenesis of lung and other cancers," Oncogene, vol. 21, no. 45, pp. 69156935, 2002.

[56] L. Prudkin, C. Behrens, D. D. Liu et al., "Loss and reduction of Fus1 protein expression is a frequent phenomenon in the pathogenesis of lung cancer," Clinical Cancer Research, vol. 14, no. 1, pp. 41-47, 2008.

[57] M. Kondo, L. Ji, C. Kamibayashi et al., "Overexpression of candidate tumor suppressor gene FUS1 isolated from the 3 p21.3 homozygous deletion region leads to G1 arrest and growth inhibition of lung cancer cells," Oncogene, vol. 20, no. 43, pp. 6258-6262, 2001.

[58] L. Ji and J. A. Roth, "Tumor suppressor FUS1 signaling pathway," Journal of Thoracic Oncology, vol. 3, no. 4, pp. 327330, 2008.

[59] L. Ji, M. Nishizaki, B. Gao et al., "Expression of several genes in the human chromosome 3p21.3 homozygous deletion region by an adenovirus vector results in tumor suppressor activities in vitro and in vivo," Cancer Research, vol. 62, no. 9, pp. 27152720, 2002.

[60] Y. P. Sher, T. F. Tzeng, S. F. Kan et al., "Cancer targeted gene therapy of BikDD inhibits orthotopic lung cancer growth and improves long-term survival," Oncogene, vol. 28, no. 37, pp. 3286-3295, 2009.

[61] M. C. Hung, G. N. Hortobagyi, and N. T. Ueno, "Development of clinical trial of E1A gene therapy targeting HER-2/neuoverexpressing breast and ovarian cancer," Advances in Experimental Medicine and Biology, vol. 465, pp. 171-180, 2000.

[62] G. N. Hortobagyi, N. T. Ueno, W. Xia et al., "Cationic liposome-mediated E1A gene transfer to human breast and ovarian cancer cells and its biologic effects: a phase I clinical trial," Journal of Clinical Oncology, vol. 19, no. 14, pp. 34223433, 2001.

[63] A. G. Cuenca, H. Jiang, S. N. Hochwald, M. Delano, W. G. Cance, and S. R. Grobmyer, "Emerging implications of nanotechnology on cancer diagnostics and therapeutics," Cancer, vol. 107, no. 3, pp. 459-466, 2006.

[64] T. Kubik, K. Bogunia-Kubik, and M. Sugisaka, "Nanotechnology on duty in medical applications," Current Pharmaceutical Biotechnology, vol. 6, no. 1, pp. 17-33, 2005.

[65] M. V. Yezhelyev, X. Gao, Y. Xing, A. Al-Hajj, S. Nie, and R. M. O'Regan, "Emerging use of nanoparticles in diagnosis and treatment of breast cancer," The Lancet Oncology, vol. 7, no. 8, pp. 657-667, 2006.

[66] E. Fattal, P. Couvreur, and C. Dubernet, "'Smart" delivery of antisense oligonucleotides by anionic $\mathrm{pH}$-sensitive liposomes," Advanced Drug Delivery Reviews, vol. 56, no. 7, pp. 931-946, 2004.

[67] S. Y. Wu and N. A.J. McMillan, "Lipidic systems for in vivo siRNA delivery," The AAPS Journal, vol. 11, no. 4, pp. 639-652, 2009. 

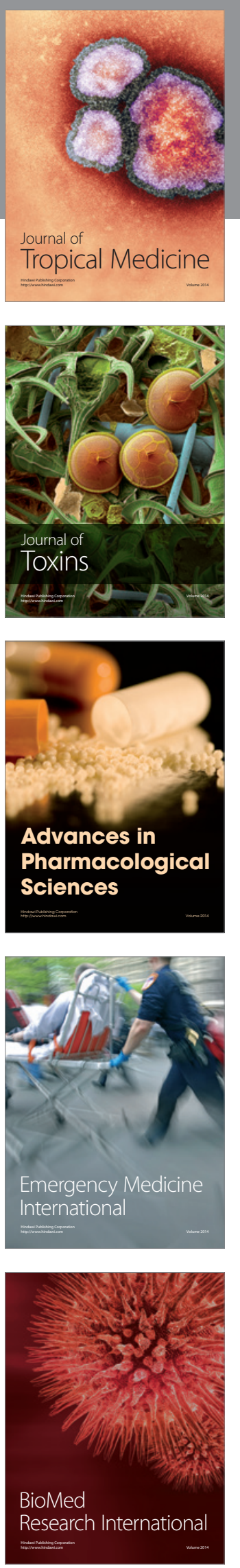
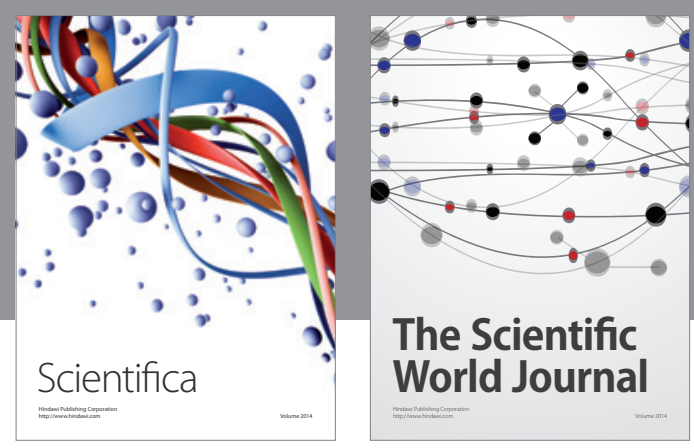

The Scientific World Journal
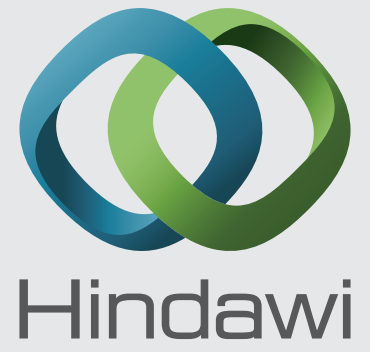

Submit your manuscripts at

http://www.hindawi.com
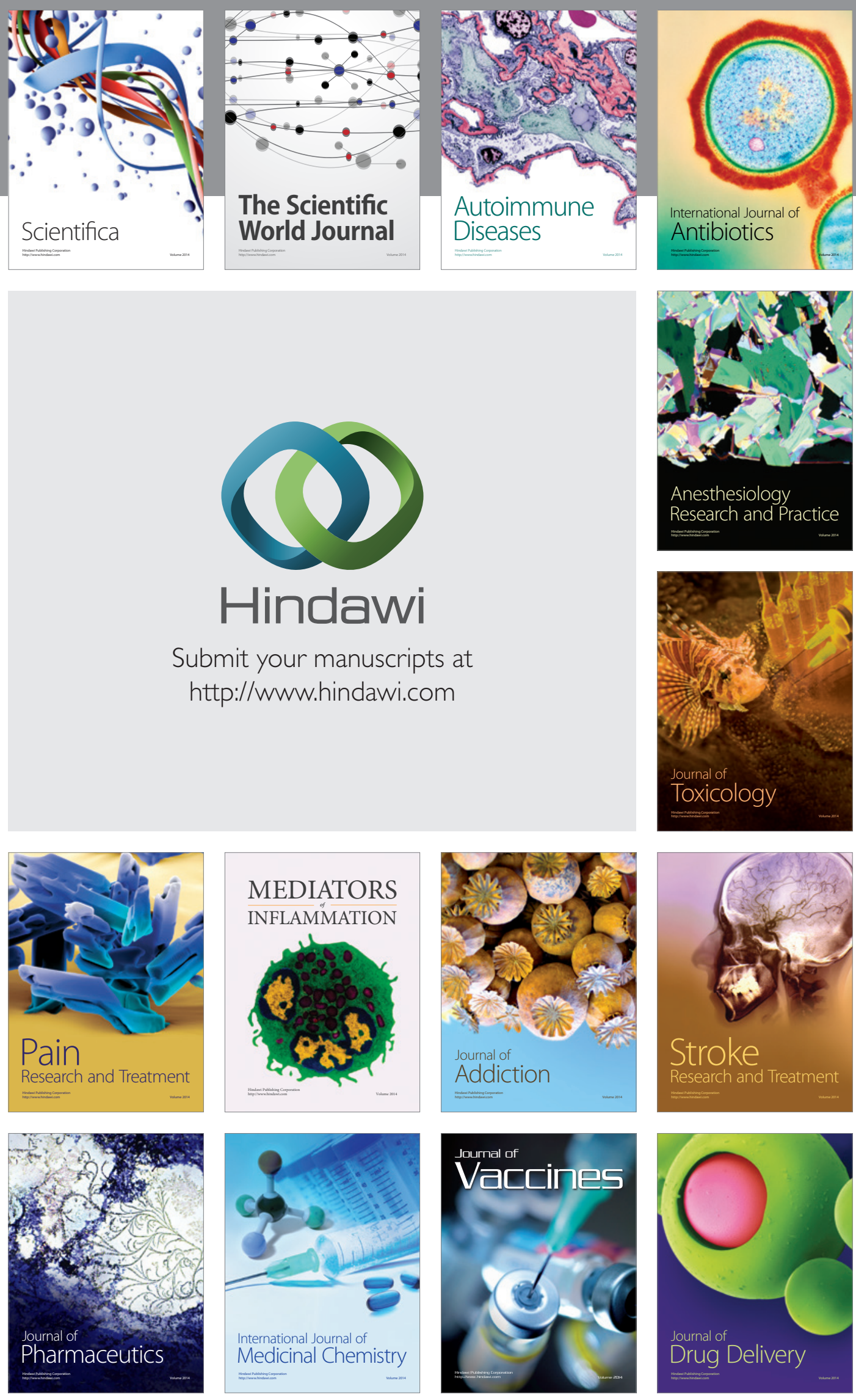